\title{
Object Manipulations in VR Show Task- and Object-Dependent Modulation of Motor Patterns
}

\author{
Jaime Maldonado \\ Cognitive Neuroinformatics, University of Bremen \\ Bremen, Germany \\ jmaldonado@uni-bremen.de
}

\author{
Christoph Zetzsche \\ Cognitive Neuroinformatics, University of Bremen \\ Bremen, Germany \\ zetzsche@informatik.uni-bremen.de
}

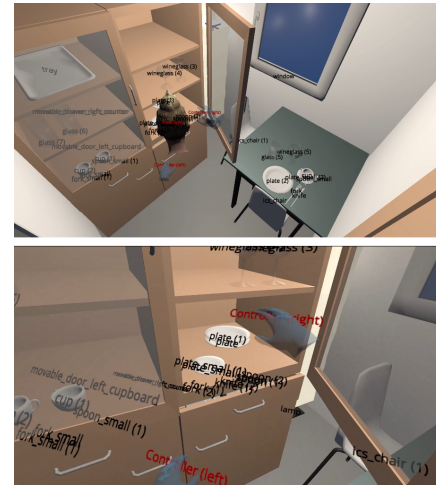

(a) Reach

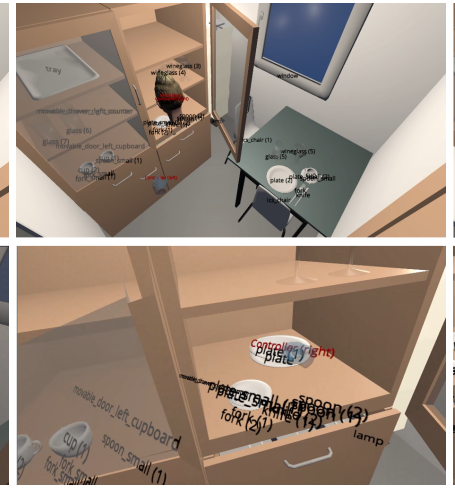

(b) Grasp

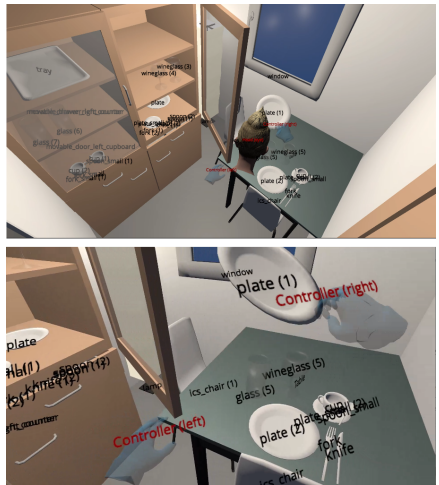

(c) Transport

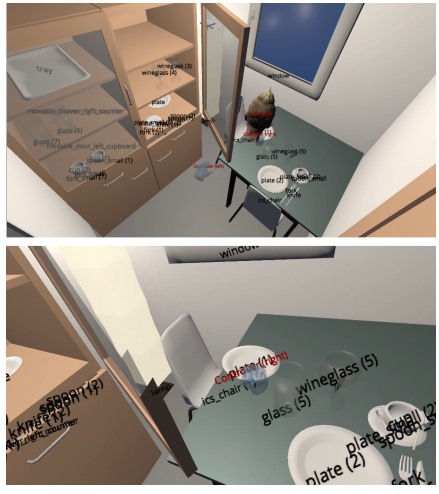

(d) Place

Figure 1: Phases of object manipulation taken from the set the table task of the HAVE dataset [21, 26]. In order to perform the task, participants took objects from the cupboard and placed them on the table. The images were extracted from the first- and third-person videos (top and bottom row, respectively) with labeled objects provided with the dataset, (Constantin Uhde.

\begin{abstract}
Humans can perform object manipulations in VR in spite of missing haptic and acoustic information. Whether their movements under these artificial conditions do still rely on motor programs based on natural experience or are impoverished due to the restrictions imposed by VR is unclear. We investigated whether reach-to-place and reach-to-grasp movements in VR can still be adapted to the task and to the specific properties of the objects being handled, or whether they reflect a stereotypic, task- and object-independent motor program. We analyzed reach-to-grasp and reach-to-place movements from participants performing an unconstrained "set-thetable" task involving a variety of different objects in virtual reality. These actions were compared based on their kinematic features. We encountered significant differences in peak speed and the duration of the deceleration phase which are modulated depending on the action and on the manipulated object. The flexibility of natural human sensorimotor control thus is at least partially transferred and exploited in impoverished VR conditions. We discuss possible explanations of this behavior and the implications for the design of object manipulations in VR.
\end{abstract}

This work is licensed under a Creative Commons Attribution-NonCommercia International 4.0 License.

VRST '21, December 8-10, 2021, Osaka, Japan

(c) 2021 Copyright held by the owner/author(s).

ACM ISBN 978-1-4503-9092-7/21/12.

https://doi.org/10.1145/3489849.3489858

\section{CCS CONCEPTS}

- Human-centered computing $\rightarrow$ Heuristic evaluations; Virtual reality; Empirical studies in $\mathrm{HCI}$.

\section{KEYWORDS}

Virtual reality, kinematics, motor control, motor skill, object manipulation phases, reach and place movements

\section{ACM Reference Format:}

Jaime Maldonado and Christoph Zetzsche. 2021. Object Manipulations in VR Show Task- and Object-Dependent Modulation of Motor Patterns. In 27th ACM Symposium on Virtual Reality Software and Technology (VRST '21), December 8-10, 2021, Osaka, Japan. ACM, New York, NY, USA, 9 pages. https://doi.org/10.1145/3489849.3489858

\section{INTRODUCTION}

Advances in VR technology enable an increasingly realism during the manipulation of virtual objects resulting from the rendering of realistic images and physics simulation. However, object manipulation in virtual and physical environments differ on the sensory information perceived by the subject. Typically, contact events in VR are perceived only in the visual modality due to the lack of haptic and acoustic rendering. Thus, motor control during task execution depends on vision and proprioception.

Considering the different phases of object manipulation (reach, grasp, transport and place, illustrated in Figure 1), the main differences between virtual and physical environments are related to the 
lack of force feedback. In VR there is no haptic feedback indicating the contact of the digits with the object. During the transport phase, the force feedback related to the object's inertia is missing. Furthermore, there is no haptic feedback indicating the contact of the object with surfaces or other objects.

In physical environments, the sensorimotor features of transport and place movements depend on the object's characteristics (size, weight, shape) and its dynamics. By contrast, the manipulation of virtual objects produces similar sensorimotor feedback, regardless of the object type or its dimensions, since transport and place movements are executed "in the air". Furthermore, reach and place movements in VR are executed under the same physical conditions, either by hands moving in free in the space or holding controls for interaction.

Given that reach and place movements in VR are executed under the same physical conditions, it could be hypothesized that there are no differences between reach-to-grasp and reach-to-place movements. On the contrary, significant differences would provide evidence that persons adjust the motor output as function of the manipulated object and the task at hand. Thus, in this paper we investigated the differences in the kinematic parameters of reach-to-place and reach-to-grasp movements executed in VR and determined whether these differences depend on the manipulated object.

We analyzed reach and place movements executed by several participants available in the set the table task of the Household Activities from Virtual Environments (HAVE) dataset [21, 26]. In contrast to studies investigating movement kinematics in VR in which movements are constrained and subjects are provided specific tasks instructions (see Section 2.3), the manipulations available in the dataset enabled us to analyze realistic movements in VR. To the best of our knowledge, no other study have been devoted to compare this kind of unconstrained reach and place movements. We found significant differences in the kinematics of reach-to-grasp and reach-to-place movements indicating that motor execution in VR is modulated by cognitive factors depending on the action and the manipulated object.

\section{RELATED WORK AND BACKGROUND}

In this section we provide a summary of the model proposed by Flanagan et al. [8], which describes the role of sensory events during the action phases of object manipulation. This is followed by a review of the main kinematic characteristics of arm movements during object manipulations performed by healthy subjects in physical and immersive virtual environments.

\subsection{Sensorimotor Control During Manipulation Tasks}

The generation of motor commands during the execution of goaldirected arm and hand movements is influenced by the following factors [6]: 1) mechanical (e.g. the degrees of freedom of the limb), 2) functional (i.e. depending on the objective of the movement, like bringing the limb to a target position), 3) cognitive (e.g. for highlevel control of the hand displacement during drawing or writing), and 4) economical (i.e. factors related to an optimization criteria, like jerk or torque minimization).
According to the influential model proposed by Flanagan et al., object manipulation can be modeled as sequence of action phases [8]. In this model, action phases are delimited by sensory events in the visual, haptic and acoustic modalities, which result from the making (or breaking) contact of the fingertips and the object and/or the contact of the object in hand with other objects or surfaces. For example, the task of grasping an object, lifting it and placing it can be divided into the following action phases: reach, load, transport, and unload. During the reach phase, the hand approaches the object and ends when the digits make contact. The load phase ends when the grasped object breaks contact with the support surface. The object is transported and the hand reaches the desired location to place the object. The transport phase ends when the object contacts the support surface. During the unload phase the object is placed and its weight is released. This phase ends when the digits release the object.

Intrinsic (e.g. size, weight and type) and extrinsic (e.g. distance and placement) object properties affect reach-to-grasp and grasp kinematics during object manipulation [7]. An analysis of the modulation of hand posture (i.e. the change of the fingers' position) during the reach movement revealed that the hand gradually changes its configuration until it reaches a final posture, which depends on the object to be grasped and the intended action to be executed after the grasp [22]. Meta-analyses show that changes in intrinsic object properties produce a large significant effect on the percentage of time spent decelerating, as well as small to medium significant effects on the movement time, peak velocity, deceleration time and the percentage of time to peak velocity [7]. Furthermore, changes in extrinsic properties show a large effect in movement time and peak velocity [7].

Sensory feedback provides information about object properties (e.g. size and weight) and about mechanical events (e.g. the contact of the fingertips with an object) necessary to update the internal representation of an object and its state [17]. The sensory feedback produced during each action phase is crucial to the sensorimotor control since it enables the monitoring of task performance [8, 17]. If an error or something unexpected occurs the motor system can react accordingly. Furthermore, sensory events provide predictions of the sensory consequences of the subsequent action phases exploiting previously learned sensorimotor correlations $[8,10]$.

The interplay between sensory feedback and motor control can be exemplified by the force grip adjustments necessary for stable grasps during object manipulation. Experimental evidence shows that sensory feedback enables the temporal coupling between grip force and the movement-induced load fluctuations during movements of a hand-held mass, where grip force is adjusted in a precisely timed manner to compensate for the peak load forces which occur during the moments of maximum acceleration and deceleration [17].

\subsection{Velocity Profiles of Arm Actions}

Stereotypical discrete (i.e. point-to-point) arm movements of healthy persons are characterized by single-peaked bell-shaped speed profiles $[15,16]$ with smooth kinematics [2]. These characteristics can be found both in planar and spatial movements [1]. 
During task performance, speed and acceleration depend on the physical characteristics of the arm and the manipulated object. In addition to the dependency of kinematics parameters on the object's characteristics described in section 2.1, the speed profile also depends on the required accuracy, whereby speed is reduced to increase accuracy (speed-accuracy trade-off) [3]. Symmetry deviations in the bell-shape profile (i.e. different duration of the acceleration and deceleration phases) have been reported for up and down movements under different speed and load conditions [18]. Thus, the velocity profile contains useful information for analyzing skilled movements [16].

Experimental evidence suggests that the kinematics of reach-tograsp movements provide information about the intention of the action to be executed with the grasped object [5, 7]. Cavallo et al. analyzed hand and wrist kinematic features of reach movements executed to grasp a bottle with two different intentions: grasp-to-pour and grasp-to-drink [5]. The analysis of the kinematic parameters showed statistically significant effects on intention. Furthermore, an analysis of the kinematic features for binary intention classification (i.e. intention to pour or to drink) showed that the wrist height and the wrist horizontal trajectory features had the highest prediction importance. A meta-analysis of experiments investigating the kinematics of reach-to-grasp movements reveals that changes in prior intentions (e.g. lift, place, hold or throw) showed small significant effects on movement time and peak velocity and a large significant effect on the percentage of time spent decelerating, which corresponds to the time after peak velocity [7]. It is important to note that the most common intention found in the meta-analysis is throw [7].

\subsection{Hand-Object Interaction in Virtual and Physical Environments}

Magdalonet et al. [14] investigated the differences of reach-to-grasp movements executed in virtual and physical environments. The experimental setup consisted of a cyberglove/grasp system for haptic feedback and a head-mounted display. The virtual and physical environments, as well as the manipulated objects, were equivalent. Subjects were requested to reach and grasp three different objects: a can, a screwdriver and a pen. The experimental results showed similar trajectories of the reach-to-grasp movement between physical and virtual environments. Regarding the movement kinematics, movements in the virtual environment were slower and had longer deceleration times. Whereas the analysis of the trajectories suggest a high level of similarity between object manipulations in virtual and physical environments, the authors also point at the influence of the interaction device used in VR on movement kinematics.

In the context of VR for rehabilitation, Furmanek et al. [9] investigated the differences between hand-object interactions performed in a haptic-free virtual environment and an equivalent physical environment. Participants were instructed to grasp three different sized rectangular objects. The virtual environment was displayed via a head-mounted display. The comparison of the reach-to-grasp movements performed in the virtual and physical environments was based on the peak velocity and the time to peak velocity of the wrist. In line with the results reported in [14], movement speed was slower in the virtual environment. With respect to the velocity profiles, the final phase of reach-to-grasp movement was longer in the virtual environment.

Cai et al. [4] compared reach and grasp movements executed in VR under different visual feedback conditions. Subjects were requested to grasp a cylinder and the virtual environment was displayed via a head mounted display. Three types of visual feedback were compared: 1) no virtual hand and no virtual cylinder, 2) virtual cylinder without virtual hand, and 3) virtual hand and cylinder. The kinematics of the reach-to-grasp movement were analyzed based on the peak wrist velocity and the time to peak wrist velocity (measured as a percentage of total movement duration). The experimental results showed no differences both in the peak velocity and the time to peak wrist velocity between the feedback conditions.

\section{MATERIALS AND METHODS}

\subsection{HAVE Dataset}

The HAVE dataset ${ }^{1}$ contains demonstrations of participants performing household tasks in 3 different virtual scenarios: 1) setting a table, 2) washing dishes, and 3) cleaning a living room. Participants were in the range from 16 to 70 years old [21]. No further demographic information is provided with the dataset. All participants were new to the scenarios [26].

Participants used a HTC Vive head mounted display and manipulated the objects with hand-held controllers. Semi-transparent hands were displayed in the position of the hand-held controllers. Objects were grasped/released by pressing/releasing the controller's grip button. The hands were animated with a simple open/close movement for the grasp/release events.

In this work we analyzed the recordings corresponding to the set the table task, which consists of demonstrations performed by 83 participants. We chose this set due to the large amount of object manipulations with different household objects involving reachto-grasp, transport and reach-to-place movements. The scenario consisted of square room $(2 \times 2 \mathrm{~m})$ containing a table, 2 chairs and a cupboard with different household objects: a tray, glasses, wineglasses, cups, plates and cutlery (forks, knifes and spoons). The virtual scenario is shown in Figure 1.

Participants received the simple instruction "please set the table". Thus, in order to perform the task participants needed to take objects out of the cupboard and place them on the table. The dataset contains first- and third-person videos with floating object labels, object and participant trajectories, and annotations of the following object manipulation events: reach, grasp, move, release and idle. The annotations include the begin and end time for each event, the hand used and the affected object. The grasp and release events were directly recorded when the controller button was pressed and released. The reach, move and idle events were automatically annotated grounded on the object grasp and release events [26].

Due to the spatial configuration and the dimensions of the virtual environment participants were able set the table without virtual locomotion, which ensures the spatial continuity of the position data. The position $(\mathrm{x}, \mathrm{y}, \mathrm{z})$ of the participants' hands (that is, the position of the hand-held controllers), as well as the position of all

${ }^{1}$ The dataset is available at https://github.com/TUM-ICS/HAVE-Dataset 
the objects in the virtual scene were recorded with a sampling rate of $90 \mathrm{~Hz}$.

The dataset aimed to provide a large number of variations of action sequences for executions of the same task [21]. This is reflected in the general instruction given to the participants. Thus, the dataset contains a large variability with respect to the objects used, the spatial configuration of the objects on the table, the number of objects manipulated (e.g. some participants set the table for one person and others for two) and the order in which objects were manipulated [21]. Thanks to the availability of position data and the large number of annotated object manipulations the dataset provides suitable information for the analysis of movement kinematics during unconstrained object manipulations in VR.

\subsection{Data Processing and Identification of Reach-to-Grasp and Reach-to-Place Movements}

The hand position data were preprocessed using a 4th order twoway low-pass Butterworth filter. The cutoff frequency was determined for each participant using residual analysis [27, 28]. Subsequently, the hand speed was computed from the filtered position data.

The automatically generated event annotations provided with the dataset comprise sequences of submovements. For example, a move event contains all the transport submovements of the object from the cupboard to the table including the final reach-to-place segment. This is illustrated with different examples in Figure 2. Regarding the reach-to-grasp movement, we noticed that for some manipulations the annotated reach event didn't include the actual reach movement (see panels a and c in Figure 2).

In order to identify the reach-to-grasp and reach-to-place movements we segmented the hand trajectories into submovements (i.e. point-to-point movements in a particular direction) and idle intervals (i.e. intervals of very low speed and non-significant displacement) by identifying the changes in the direction of movement and the movement onsets/offsets based on the hand speed. We located the submovement previous to the start of the annotated grasp event and identified it as the reach-to-grasp movement. Similarly, we identified the reach-to-place movement as the submovement previous to the start of the annotated release event. Different examples of the event annotations provided with the dataset, as well as the identified reach-to-grasp and reach-to-place movements during the manipulation of different objects are shown in Figure 2.

\subsection{Data Analysis}

We analyzed data from 82 out of the 83 participants performing the set the table task (the event annotations for the participant identifier 29 were not available). We will refer to a sequence of reach-to-grasp, grasp, transport, reach-to-place, and place actions as a manipulation. In this work we analyzed the manipulation of transportable objects.

We left out the analysis the chair and tray objects due to the low number of manipulations. We also left out of the analysis manipulations in which participants adjusted the position of objects already placed on the table due to the following reasons: 1) the manipulation required small-amplitude movements, and 2) the placing required a much finer motor control to perform small position adjustments. Finally, we didn't include left hand manipulations due their small number compared to those performed with the right hand. The number of analyzed manipulations per object was distributed follows (object-number): cup-103, cutlery-318, glass-113, plate-193, and wineglass-110. From each manipulation we analyzed the reach-to-grasp and reach-to-place actions. Thus, we analyzed 837 reach-to-grasp and 837 reach-to-place actions in total.

Kinematic features were computed to compare the characteristics of the reach-to-grasp and reach-to-place movements. The peak speed (PS) provides an indirect measure of the speed-accuracy tradeoff associated with the movement, where lower speeds indicates a more accurate and careful behavior. Additionally, we measure the symmetry of the speed profile by computing the proportion of time to peak speed (PTPS), that is, the time to peak speed over the total movement duration. PTPS values below 0.5 indicate a longer deceleration phase.

\subsection{Statistical Modeling}

To assess the differences between reach-to-place and reach-to-grasp movements, and to determine whether these differences depend on the manipulated object, the kinematic parameters were analyzed using generalized linear mixed-effects models (GLMMs) ${ }^{2}$. This type of statistical model allows for analyzing the kinematic parameters with unbalanced number of actions per participant and object, without aggregating the data over object types or participants [24]. The modeling and the statistical analyses were performed with $\mathrm{R}$ [20]. The GLMMs were fitted using the afex package [23] with the likelihood ratio tests (LRT) method. Differences between actions and objects, as well as pairwise comparisons on the fitted models, were analyzed based on the estimated marginal means with asymptotic degrees of freedom using the emmeans package [12]. The R notation for the specification of the GLMMs and the formulas of the fitted models are included in the Appendix A.

A GLMM requires the specification of 1) fixed factors, which are the explanatory factors responsible for the systematic variation in the dependent variables, 2) the random factors, which model the sampling structure contributing to random variability in the dependent variables, and 3) the dependencies between the fixed and the random factors $[13,24]$.

In our model the fixed factors are the action and the manipulated object. Action is a within-participants factor with two levels: reach-to-grasp and reach-to-place. Object is a between-participants factor (not all participants manipulated every object) with five levels: cup, cutlery, glass, plate, and wineglass. We set the participant as random factor. This enables us to determine the extent to which mean responses vary across participants and reduce the Type I error rate by considering the variability in the dependent variables that are associated with each participant rather than the fixed factors [13]. The specification of random factors aims to model the idiosyncrasies of the sample [24]. Thus, the model captures the variability introduced by each participant. For example, some participants might be slower than the average.

The GLMM specifies the main effects and interaction of the action and object fixed factors. For the specification of the random effects

\footnotetext{
${ }^{2}$ For an introduction to this class of statistical models for the analysis of repeated measures data see [24].
} 
Cup manipulation

Position with original automatic action annotation (action hand)

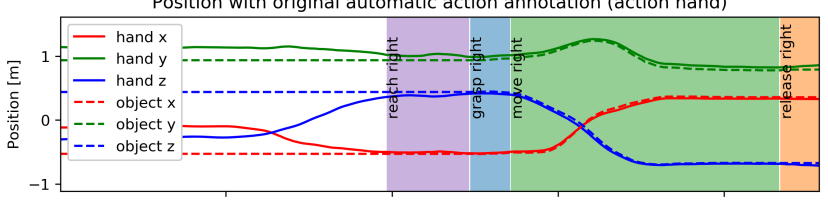

Speed with new action annotations (action hand)

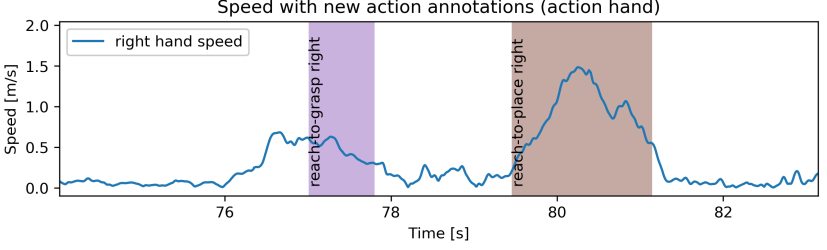

(a) The top panel shows that the orginal reach annotation doesn't include the actual reach-to-grasp movement. Instead it contains an idle interval of very low speed with non-significant displacement as observed in the $\mathrm{x}, \mathrm{y}$ and $\mathrm{z}$ traces. The reach-to-place movement occured immediately after the grasp and was followed by an idle interval.

Spoon manipulation

Position with original automatic action annotation (action hand)

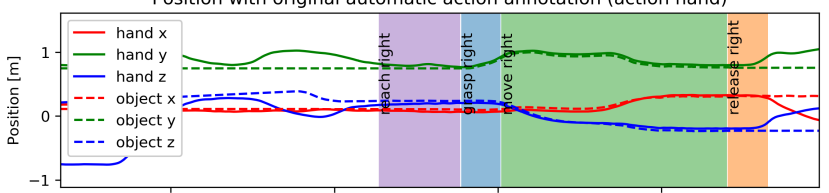

Speed with new action annotations (action hand)

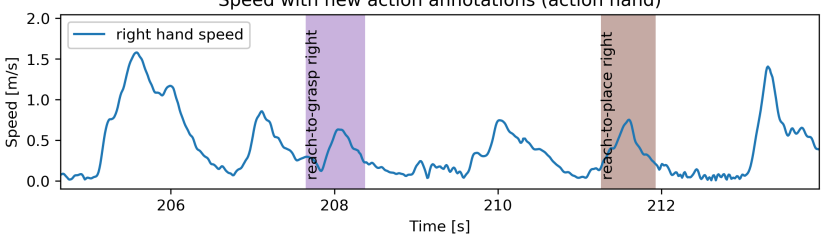

(c) The top panel shows that the orginal reach annotation doesn't include the actual reach-to-grasp movement. The actual reach-to-place movement occured approximately in the middle of the originally annotated move action.
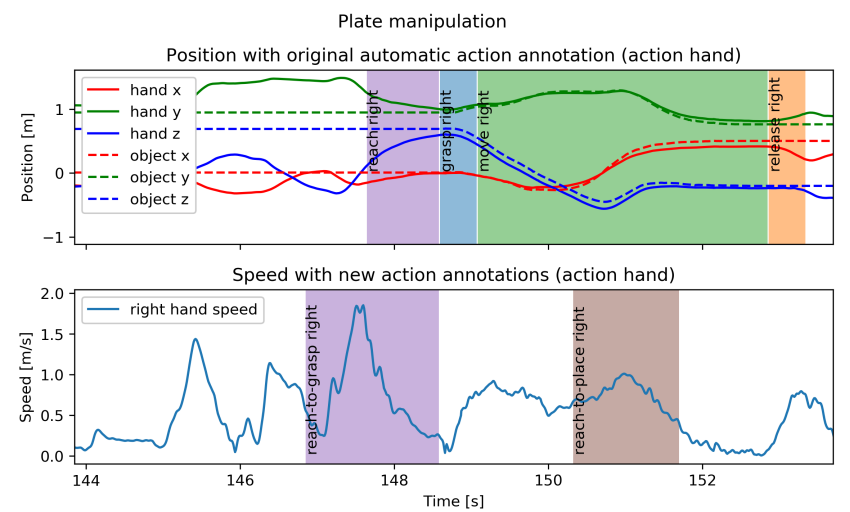

(b) The top panel shows that the original annotation doesn't include the complete reach-to-grasp movement. This is corrected in the new annotation. The top panel also shows how the original move annotation contains a submovement, the actual reach-to-place movement, and an idle interval.

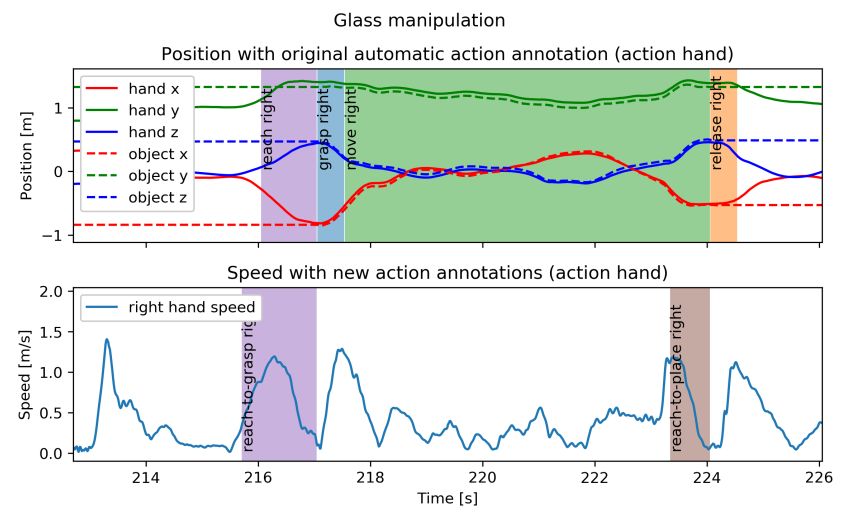

(d) This example shows a good match between the original reach and the new reach-tograsp annotation. In contrast to the other examples, here the reach-to-place movement occured at the end of the originally annotated move action.

Figure 2: Manipulation examples of different objects showing the hand and object position, as well as the hand's speed profile. The examples illustrate the difference between the action annotations provided with the dataset and the identified reach-tograsp and reach-to-place actions. The examples were extracted from the participant identifier 61.

structure we took into account that the size of the differences in the kinematic parameters between actions might differ between participants. This was modeled as a random slope of action within participant with correlated intercept. Additionally, we considered the fact that not all the objects were manipulated by each participant. This was modeled as a random variation in intercept among objects within participants.

Using this specification we fitted two models, one for PS and one for PTPS. The model fitted for PTPS produced a singular fit warning, which indicates that the parameters associated to the random factors cannot be fully identified. Following the recommendation given in [24], we removed the correlation among random slopes of action within participant and refitted the PTPS model.

\section{RESULTS}

The distribution of PS values measured per action and object are shown in Figure 3. A mixed model ANOVA test (Type 3, LRTmethod, see Table 1) reveals significant main effects for action $\left(\chi^{2}(1)=18.69, p<.001\right)$ and a significant interaction of action by object $\left(\chi^{2}(4)=27.07, p<.001\right)$. Post-hoc pairwise comparisons (Holm corrected, see Table 2) reveal a significant difference in PS between actions for the following objects: glass $(p<.0001)$, plate $(p<.0001)$, and wineglass $(p=.003)$. These results indicate that, in general, reach-to-grasp tend to be faster than reach-to-place movements. However, significant differences were only observed for the glass, plate and wineglass objects. This suggest that these differences are object-dependent.

The distribution of PTPS values measured per action and object are shown in Figure 4. A mixed model ANOVA test (Type 3, LRT-method, see Table 3) reveals significant main effects for object 


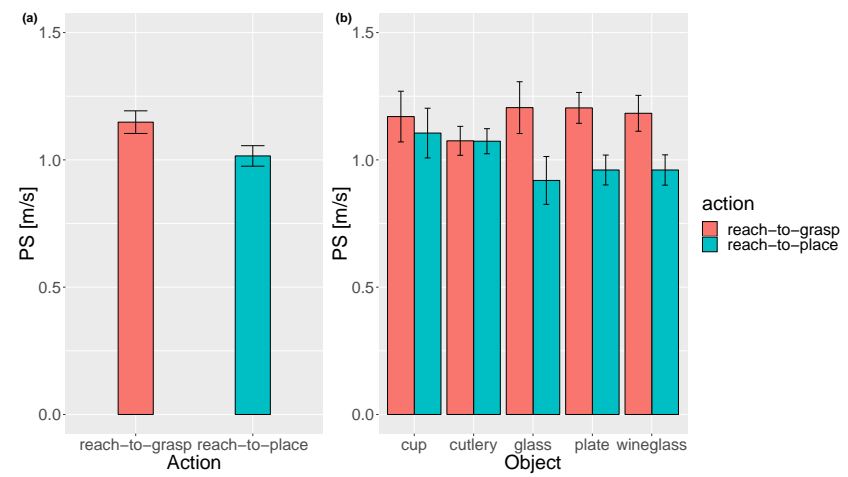

Figure 3: Peak speed (a) per action and (b) per action and object. The error bars represent the $95 \%$ confidence interval for the mean.

Table 1: Mixed Model Anova Table (Type 3 tests, LRTmethod) for dependent variable PS

\begin{tabular}{lrll}
\hline Effect & df & $\chi^{2}$ & p.value \\
\hline action & 1.00 & $18.69^{* * *}$ & $<.001$ \\
object & 4.00 & 5.06 & .281 \\
action:object & 4.00 & $27.07^{* * *}$ & $<.001$ \\
\hline
\end{tabular}

$\left(\chi^{2}(4)=25.97, p<.001\right)$. Post-hoc pairwise comparisons (Holm corrected, see Table 4) reveal significant differences in PTPS between the following object pairs: cup-cutlery $(p=.0035)$, cup-plate ( $p=.0012)$, cup-wineglass $(p<.0001)$. All PTPS values per object were less than $0.5-$ cup: $t(205)=-3.4403, p=.000352$; cutlery: $t(635)=-13.388 p<.0001$; glass: $t(225)=-8.2278, p<.0001 ;$ plate: $t(385)=-10.385, p<.0001$; wineglass: $t(219)=-11.358$, $p<.0001-$ indicating that for all objects the reach-to-grasp and reach-to-place movements tend to be asymmetric with a longer deceleration phase (i.e. PTPS < 0.5).
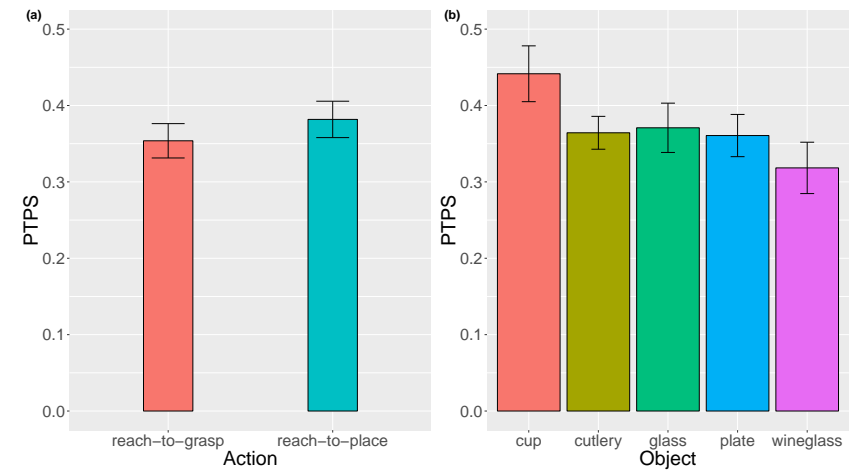

Figure 4: Proportion of time to peak speed (a) per action and (b) per object. The error bars represent the $95 \%$ confidence interval for the mean.

\section{CONCLUSIONS AND DISCUSSION}

In this paper we investigated the differences between reach-tograsp and reach-to-place movements executed in VR. Manipulations performed by 82 participants were analyzed. We identified submovements within the hand trajectories and identified the reachto-grasp and reach-to-place actions. The kinematics of each action were analyzed based on the PS and PTPS computed from the speed profiles. These parameters indicate how fast an action is performed and whether the velocity profile is asymmetric. Experimental evidence on the manipulation in physical environments shows that these parameters are modulated by intention, as well as by intrinsic and extrinsic object properties during reach-to-grasp movements [7]. In addition they are related to the skill with which a movement is executed, since the speed profile of adults depends on the required accuracy [3].

Before going into a detailed interpretation of our results it is important to note that the VR setup is completely "neutral" with respect to the parameters. Due to the characteristics of the hand-held interaction device, the object-dependent modulation of finger position previous to the grasp that occurs during physical manipulation [22] is not present in the HAVE dataset. Consequently, the different virtual objects were manipulated with the same physical grasp. Additionally, load force fluctuations and grip force adjustments executed close to movement onset and offset [17] do not resemble those that would occur during the manipulation of different physical objects. In physical environments, objects like plates and cutlery behave with different dynamics. In contrast, the load force fluctuations experienced by the participants in VR, like those perceived as the hand approaches the table during the reach-to-place movement, are the same regardless of the grasped virtual object. Furthermore, both object grasp and release required a button trigger. Considering these factors, all movements performed in VR and the associated feedback information up to the moment of contact are completely equivalent for a reach-to-grasp and a reach-to-place action, and they do also not depend on the objects, since the VR behavior is completely identical for all different objects. Thus, if a subject would act optimally with respect to the VR situation, it would use one-and-the same velocity profile for the reach-to-grasp and for the reach-to-place action invariably for all objects.

Now let us consider what our subjects are doing: significant differences in PS indicate that reach-to-grasp movements were typically faster than the reach-to-place movements. This is in line with the results reported in [19] for healthy control subjects manipulating a cylindrical object in which reach-to-grasp movements were faster than reach-to-place movements (termed transport-to-place). Interestingly, we also encountered a significant interaction of action by object for the glass, plate and wineglass objects. This indicates that the reach-to-place phase, at least for these latter objects, was performed with slower movements. This is also associated with a softer deceleration and a more accurate movement. This makes sense in the real world, since here glasses and wineglasses are fragile objects which might easily break if the final velocity at the point of contact is too high. Cups, and in particular cutlery, are much more robust with respect to final contact forces. Only the plate seems to stick out here, because it will usually not be more delicate than a cup. But plates are much larger objects than all the other 
Table 2: Pairwise comparisons of PS for the significant interaction of action by object.

\begin{tabular}{llrrrrl}
\hline contrast & object & estimate & SE & df & z.ratio & p.value \\
\hline (reach-to-grasp) - (reach-to-place) & cup & 0.0644 & 0.0673 & $\mathrm{Inf}$ & 0.956 & .6778 \\
(reach-to-grasp) - (reach-to-place) & cutlery & 0.0146 & 0.0448 & $\mathrm{Inf}$ & 0.327 & .7439 \\
(reach-to-grasp) - (reach-to-place) & glass & 0.2937 & 0.0643 & $\mathrm{Inf}$ & 4.566 & $<.0001$ \\
(reach-to-grasp) - (reach-to-place) & plate & 0.2354 & 0.0523 & $\mathrm{Inf}$ & 4.505 & $<.0001$ \\
(reach-to-grasp) - (reach-to-place) & wineglass & 0.2130 & 0.0647 & $\mathrm{Inf}$ & 3.292 & .0030 \\
\hline
\end{tabular}

Degrees-of-freedom method: asymptotic

$P$ value adjustment: Holm method for 5 tests

Table 3: Mixed Model Anova Table (Type 3 tests, LRTmethod) for dependent variable PTPS

\begin{tabular}{lrll}
\hline Effect & df & $\chi^{2}$ & p.value \\
\hline action & 1.00 & 2.49 & .115 \\
object & 4.00 & $25.977^{* * *}$ & $<.001$ \\
action:object & 4.00 & 6.97 & .137 \\
\hline
\end{tabular}

Table 4: Pairwise comparisons of PTPS for the significant main effects for object.

\begin{tabular}{lrrrrl}
\hline contrast & estimate & SE & df & z.ratio & p.value \\
\hline cup - cutlery & 0.0757 & 0.0215 & $\operatorname{Inf}$ & 3.518 & .0035 \\
cup - glass & 0.0669 & 0.0251 & $\operatorname{Inf}$ & 2.670 & .0531 \\
cup - plate & 0.0858 & 0.0225 & $\operatorname{Inf}$ & 3.812 & .0012 \\
cup - wineglass & 0.1259 & 0.0251 & $\operatorname{Inf}$ & 5.016 & $<.0001$ \\
cutlery - glass & -0.0088 & 0.0205 & $\operatorname{Inf}$ & -0.430 & 1.0000 \\
cutlery - plate & 0.0100 & 0.0175 & $\operatorname{Inf}$ & 0.574 & 1.0000 \\
cutlery - wineglass & 0.0501 & 0.0206 & $\operatorname{Inf}$ & 2.436 & .0892 \\
glass - plate & 0.0188 & 0.0218 & $\operatorname{Inf}$ & 0.862 & 1.0000 \\
glass - wineglass & 0.0589 & 0.0244 & $\operatorname{Inf}$ & 2.415 & .0892 \\
plate - wineglass & 0.0401 & 0.0219 & $\operatorname{Inf}$ & 1.835 & .2661 \\
\hline
\end{tabular}

Results are averaged over the levels of: action

Degrees-of-freedom method: asymptotic

$P$ value adjustment: Holm method for 10 tests

ones and might thus represent a greater danger of causing damage if not positioned with sufficient precision.

Overall, our analysis indicates that asymmetric behavior is a general property of unconstrained reach-to-grasp and reach-toplace movements in VR. On the object level all the PTPS values were significantly lower than 0.5 . These results indicate that both reach-to-grasp and reach-to-place movements tend to have asymmetric speed profiles with longer deceleration phases. These results are similar to those reported in [19] for healthy control subjects manipulating a cylindrical object in which the deceleration time is longer than the time to peak velocity both for reach-to-grasp and reach-to-place movements. The longer deceleration times of the reach-to-grasp movements observed in our analysis are also in line with the results reported in [14] and [9] for reach-to-grasp movements in constrained experimental setups, generalizing this observation to unconstrained setups. On the object level, we encountered significant PTPS differences between the cup and the other objects. Whether this implies that cup manipulations are performed with a different deceleration time course requires a more detailed analysis of the velocity profiles.

Due to the lack of haptic and acoustic rendering, motor control during the analyzed actions depended on vision and proprioception. In a somewhat similar situation, an experiment comparing the execution of vertical and horizontal bi-directional movements of a hand-held mass between a group of healthy control subjects and a patient who had no tactile or proprioceptive sensations showed that movements were executed with similar acceleration profiles, only differing on the timing and the magnitude of the grip force [17]. This result suggests that motor plans controlled with vision show a similar kinematic profile to those controlled with the complete sensory input (haptic, vision and proprioception). Thus, it can be expected that movements executed in VR under visual and proprioceptive control show speed profiles similar to those that would be observed during the manipulation of physical objects, regardless of the missing object-dependent load force fluctuations, haptic sensations and acoustic signals during contacts.

We observed action-object dependencies in the PS and the PTPS parameters of the speed profiles. In particular, differences on the object level indicate that the intrinsic object properties modulated motor behavior in VR. These results are thus in line with the experimental evidence observed during manipulations in physical environments where intrinsic object properties have an effect on the percentage of time spent decelerating and on peak velocities [7]. It is important to note that, due to the characteristics of the HAVE dataset, we focused our analysis on the influence of the object type and the action. Intention was not considered as an analysis factor since it was equal for all the manipulations (i.e. participants intended, as instructed, to take objects out of the cupboard and place them on the table).

Viewed as a whole, our results show that unconstrained actions in VR show characteristics of actions executed in physical environments: 1) reach-to-grasp movements faster than reach-to-place movements, and 2) asymmetric reach-to-grasp and reach-to-place movements with longer deceleration times. Furthermore, the objectdependent differences suggest that cognitive factors modulate motor output based the on object type and action. Recalling that the velocity profile without consideration of real-world constraints like the breakability of objects would be similar for all objects, a possible explanation of these results would be that participants modulated their motor behavior in VR based on previous experiences in the real world $[8,10]$, where breakable household objects like glasses 
are handled carefully and with accurate movements in order to avoid damages or spilling their content.

It is an interesting question to ask how this transfer of sensorimotor properties between the natural experience of the real-world and VR conditions takes place. Is it based on a higher-level cognitive decision and reasoning process in the style of: "I recognize this object in VR and I know it is breakable in the real world and therefore I adjust the parameters of my motor program accordingly"?. The interesting point here is that subjects will usually know that this damage would not happen in VR. So in spite of this knowledge, would they use common-world reasoning to adapt their motor program to a sub-optimal behavior? The other extreme explanation, ignoring the explanatory gray shades in between, would be that of a mandatory low-level process. The mere presence of a glass as object of manipulation, even if only signalized by vision, and never associated with breakability under VR conditions, would inevitably invoke a motor routine with reduced peak speed, asymmetric velocity profile and softer deceleration phase. In its radical version, this would imply that subjects would have difficulties in switching this towards the VR-optimized, general and object-independent velocity profile. Doubtless an interesting topic for future investigations.

Our results are suitable of being applied in the design of object manipulation rules and the simulation of agent behaviors in order to increase realism and immersiveness in virtual environments. Even if it is not possible to simulate the hand-object interaction on a detailed physical level, immersiveness could be improved if the easily measurable trajectory parameters we have identified here are used to trigger a suitable feedback. For example, measuring high peak speed combined with short deceleration time could trigger the breaking of a glass. This would encourage a more careful behavior during subsequent manipulations.

Furthermore, the characteristic human behavior observed in our analysis can inform the object manipulations performed by virtual agents, enabling them to produce a more human-like behavior than the usual inverse kinematic and optimization results. In general, it has been shown that the kinematic parameters of synthetic agents influence the perception of humanness of gait behavior [25] and the judgment of consistency of an agent's physical structure and the produced motion [11]. In particular, for the simulation of goal directed arm actions it has been shown that the kinematics of an animated human agent, together with the characteristics of its body, have a strong influence on the perceived weight consistency for pushing, lifting, and throwing actions in VR [11]. Movement trajectories generated by means of inverse kinematics could incorporate the time course of reach-to-grasp and reach-to-place movements. Apart from generating object-dependent behaviors, this would also enable the simulation of both excessively careful and ridiculously clumsy agents.

\section{ACKNOWLEDGMENTS}

The research reported in this paper has been supported by the German Research Foundation, as part of Collaborative Research Center 1320 "EASE - Everyday Activity Science and Engineering", University of Bremen (http://www.ease-crc.org/). The research was conducted in subproject H01 - Sensorimotor and Causal Human Activity Models for Cognitive Architectures. The authors would like to thank Kevin Maldonado for his valuable insights during the early phase of the manuscript preparation.

\section{REFERENCES}

[1] Satyajit Ambike and James P. Schmiedeler. 2013. Invariant geometric characteristics of spatial arm motion. Experimental Brain Research 229, 1 (jun 2013), 113-124. https://doi.org/10.1007/s00221-013-3599-9

[2] Sivakumar Balasubramanian, Alejandro Melendez-Calderon, Agnes Roby-Brami, and Etienne Burdet. 2015. On the analysis of movement smoothness. Fournal of NeuroEngineering and Rehabilitation 12, 1 (dec 2015). https://doi.org/10.1186/ s12984-015-0090-9

[3] Ettiene Burdet and Theodore E. Milner. 1998. Quantization of human motions and learning of accurate movements. Biological Cybernetics 78, 4 (may 1998), 307-318. https://doi.org/10.1007/s004220050435

[4] Qiuwen Cai, Junhua Li, and Jinyi Long. 2021. Effect of Physical and Virtual Feedback on Reach-to-Grasp Movements in Virtual Environments. IEEE Transactions on Cognitive and Developmental Systems (2021), 1-1. https://doi.org/10.1109/tcds. 2021.3066618

[5] Andrea Cavallo, Atesh Koul, Caterina Ansuini, Francesca Capozzi, and Cristina Becchio. 2016. Decoding intentions from movement kinematics. Scientific Reports 6, 1 (nov 2016). https://doi.org/10.1038/srep37036

[6] Michel Desmurget, Denis Pelisson, Yves Rossetti, and Claude Prablanc. 1998. From Eye to Hand: Planning Goal-directed Movements. Neuroscience \& Biobehavioral Reviews 22, 6 (oct 1998), 761-788. https://doi.org/10.1016/s0149-7634(98)00004-9

[7] Ida Egmose and Simo Køppe. 2017. Shaping of Reach-to-Grasp Kinematics by Intentions: A Meta-Analysis. Fournal of Motor Behavior 50, 2 (jun 2017), 155-165. https://doi.org/10.1080/00222895.2017.1327407

[8] J. Randall Flanagan, Miles C. Bowman, and Roland S. Johansson. 2006. Control strategies in object manipulation tasks. Current Opinion in Neurobiology 16, 6 (dec 2006), 650-659. https://doi.org/10.1016/j.conb.2006.10.005

[9] Mariusz P. Furmanek, Luis F. Schettino, Mathew Yarossi, Sofia Kirkman, Sergei V. Adamovich, and Eugene Tunik. 2019. Coordination of reach-to-grasp in physical and haptic-free virtual environments. Fournal of NeuroEngineering and Rehabilitation 16, 1 (jun 2019). https://doi.org/10.1186/s12984-019-0525-9

[10] Roland S. Johansson and J. Randall Flanagan. 2009. Coding and use of tactile signals from the fingertips in object manipulation tasks. Nature Reviews Neuroscience 10, 5 (apr 2009), 345-359. https://doi.org/10.1038/nrn2621

[11] Sophie Kenny, Naureen Mahmood, Claire Honda, Michael J. Black, and Nikolaus F. Troje. 2019. Perceptual Effects of Inconsistency in Human Animations. ACM Transactions on Applied Perception 16, 1 (mar 2019), 1-18. https://doi.org/10.1145/ 3301411

[12] Russell V. Lenth. 2021. emmeans: Estimated Marginal Means, aka Least-Squares Means. https://CRAN.R-project.org/package=emmeans R package version 1.6.2-1.

[13] Steson Lo and Sally Andrews. 2015. To transform or not to transform: using generalized linear mixed models to analyse reaction time data. Frontiers in Psychology 6 (aug 2015). https://doi.org/10.3389/fpsyg.2015.01171

[14] Eliane C. Magdalon, Stella M. Michaelsen, Antonio A. Quevedo, and Mindy F. Levin. 2011. Comparison of grasping movements made by healthy subjects in a 3-dimensional immersive virtual versus physical environment. Acta Psychologica 138, 1 (sep 2011), 126-134. https://doi.org/10.1016/j.actpsy.2011.05.015

[15] H. Nagasaki. 1989. Asymmetric velocity and acceleration profiles of human arm movements. Experimental Brain Research 74, 2 (1989). https://doi.org/10.1007/ bf00248865

[16] Wendy L. Nelson. 1983. Physical principles for economies of skilled movements. Biological Cybernetics 46, 2 (feb 1983), 135-147. https://doi.org/10.1007/ bf00339982

[17] Dennis A. Nowak, Stefan Glasauer, and Joachim Hermsdörfer. 2004. How predictive is grip force control in the complete absence of somatosensory feedback? Brain 127, 1 (jan 2004), 182-192. https://doi.org/10.1093/brain/awh016

[18] Charalambos Papaxanthis, Thierry Pozzo, and Paul Stapley. 1998. Effects of movement direction upon kinematic characteristics of vertical arm pointing movements in man. Neuroscience Letters 253, 2 (sep 1998), 103-106. https: //doi.org/10.1016/s0304-3940(98)00604-1

[19] Shahina Pardhan, Amy Scarfe, Rupert Bourne, and Matthew Timmis. 2017. A Comparison of Reach-to-Grasp and Transport-to-Place Performance in Participants With Age-Related Macular Degeneration and Glaucoma. Investigative Opthalmology \& Visual Science 58, 3 (mar 2017), 1560. https://doi.org/10.1167/iovs.16-20273

[20] R Core Team. 2021. R: A Language and Environment for Statistical Computing. $\mathrm{R}$ Foundation for Statistical Computing, Vienna, Austria. https://www.R-project. org/

[21] Karinne Ramirez-Amaro, Constantin Uhde, Tamas Bates, and Gordon Cheng. 2019. A benchmarking dataset for automatic symbolic grounding from virtual demonstrations. In 2nd International Workshop on Computational Models of Affordance in Robotics (ICRA). 
[22] Marco Santello, Martha Flanders, and John F. Soechting. 1998. Postural Hand Synergies for Tool Use. The fournal of Neuroscience 18, 23 (dec 1998), 10105-10115. https://doi.org/10.1523/jneurosci.18-23-10105.1998

[23] Henrik Singmann, Ben Bolker, Jake Westfall, Frederik Aust, and Mattan S. BenShachar. 2021. afex: Analysis of Factorial Experiments. https://CRAN.R-project. org/package=afex R package version $0.28-1$.

[24] Henrik Singmann and David Kellen. 2019. An Introduction to Mixed Models for Experimental Psychology. In New Methods in Cognitive Psychology. Routledge, 4-31. https://doi.org/10.4324/9780429318405-2

[25] James C. Thompson, J. Gregory Trafton, and Patrick McKnight. 2011. The Perception of Humanness from the Movements of Synthetic Agents. Perception 40, 6 (jan 2011), 695-704. https://doi.org/10.1068/p6900

[26] Constantin Uhde, Nicolas Berberich, Karinne Ramirez-Amaro, and Gordon Cheng 2020. The Robot as Scientist: Using Mental Simulation to Test Causal Hypotheses Extracted from Human Activities in Virtual Reality. In 2020 IEEE/RS7International Conference on Intelligent Robots and Systems (IROS). IEEE. https://doi.org/10. 1109/iros45743.2020.9341505

[27] David A. Winter. 2009. Biomechanics and Motor Control of Human Movement (4th ed.). John Wiley \& Sons, Inc.

[28] Bing Yu, David Gabriel, Larry Noble, and Kai-Nan An. 1999. Estimate of the Optimum Cutoff Frequency for the Butterworth Low-Pass Digital Filter. Fourna of Applied Biomechanics 15, 3 (aug 1999), 318-329. https://doi.org/10.1123/jab.15. 3.318

\section{A R NOTATION FOR THE SPECIFICATION OF THE GLMMS}

In this appendix we provide the R notation used for the specification of GLMMs and the formulas of the fitted models.

\section{A.1 Specification of main effects and interaction}

The GLMM specifies the main effects and interaction of the action and object fixed factors: action*object

\section{A.2 Specification of the random effects structure}

The GLMM specifies the random effects as a random slope of action within participant with correlated intercept:

(action/participant)

Additionally, the model includes a random variation in intercept among objects within participants:

(1|participant:object)

\section{A.3 Fitted Model for PS}

PS action*object + (action|participant) +
(1|participant:object)

\section{A.4 Fitted Model for PTPS}

The following model produced a singular fit warning: PTPS action*object + (action|participant) + (1)participant: object)

therefore, following the recommendation given in [24], we removed the correlation among random slopes of action within participant and refitted the model as follows:

PTPS action*object + (action||participant) +

(1|participant: object) 